\title{
Coulomb blockade in graphene nanoribbons
}

\author{
F. Sols ${ }^{1}$, F. Guinea ${ }^{2}$, and A. H. Castro Neto ${ }^{3}$ \\ 1 Departamento de Física de Materiales, Universidad Complutense de Madrid, E-28040 Madrid, Spain \\ ${ }^{2}$ Instituto de Ciencia de Materiales, CSIC, E-28049 Cantoblanco, Madrid, Spain and \\ 3 Physics Department, Boston University, 590 Commowealth Avenue, Boston, Massachussetts 02215, USA
}

\begin{abstract}
We propose that recent transport experiments revealing the existence of an energy gap in graphene nanoribbons may be understood in terms of Coulomb blockade. Electron interactions play a decisive role at the quantum dots which form due to the presence of necks arising from the roughness of the graphene edge. With the average transmission as the only fitting parameter, our theory shows good agreement with the experimental data.
\end{abstract}

PACS numbers: 72.10.-d, 73.23.Hk, 81.05.Uw

Graphene, a two-dimensional allotrope of carbon on a honeycomb lattice, was isolated a few years ago [1] creating a great excitement in the physics community due to its close connections to high-energy particle physics [2, 3] and its tantalizing possible technological applications [4, 5]. It is now experimentally established that a great deal of the properties of graphene [6] can be described in terms of non-interacting (or weakly interacting) linearly dispersing Dirac quasi-particles [7, 8]. The only accepted exception maybe when graphene is subject to strong magnetic fields in the quantum Hall regime [9], when the electronic kinetic energy is quenched by the appearance of Landau levels, and the fourfold degeneracy of the Landau levels is split by electron-electron interactions [10, 11]. Nevertheless, in the absence of an applied magnetic field, because of the vanishing of the density of states for Dirac fermions in two dimensions [12], the electrons in graphene interact through strong, essentially unscreened, long-range Coulomb interactions 13, 14, 15, 16]. The fact that Coulomb interactions do not show up in bulk experiments remains a puzzle in the physics of graphene.

Recent experiments on the electron transport properties of lithographically patterned graphene nanoribbons have shown the existence of an energy gap near the charge neutrality point [17]. The size of the gap $E_{g}$ is inferred from the nonlinear conductance at low temperatures and is found to decrease with the ribbon width $W$ following the approximate law $E_{g}=\alpha /\left(W-W^{*}\right)$. This result seems to correlate with a conductance behavior $G=\beta\left(W-W_{0}\right)$, since $W^{*} \approx W_{0} \simeq 16 \mathrm{~nm}$ for the same sample at temperature $T=1.6 \mathrm{~K}$. In the absence of interaction effects, the energy gaps between subbands in a graphene ribbon should scale inversely with the ribbon width, $E_{g} \approx \hbar v_{\mathrm{F}} / W$, where $v_{\mathrm{F}} \approx 10^{6} \mathrm{~m} / \mathrm{s} \approx 0.66 \mathrm{eV}$ $\times \mathrm{nm} \times \hbar^{-1}$ is the Fermi velocity in graphene [6]. Nevertheless, this estimate leads to gaps which are smaller than those observed experimentally (note that, for widths of about $20 \mathrm{~nm}$ the experimental gaps are larger than $0.1-0.2 \mathrm{eV})$. This result has led to the suggestion that the effective transport width is reduced with respect to the nominal width $W$ by an amount $W^{*} \approx W_{0}$ due to the existence of structural disorder at the edges or to a systematic inaccuracy in the determination of the geometrical width caused by over-etching beneath the etch mask [17]. On the other hand, it has been shown that graphene quantum dots as large as $25 \mu \mathrm{m}$ (at low temperatures), and as small as $40 \mathrm{~nm}$ (at room temperature), show Coulomb blockade effects [6] indicating that electron interactions become stronger as the dimensions of graphene sheets are reduced.

In the present work we argue that the main results of Ref. 17] can be naturally explained as due to Coulomb blockade effects originated by the roughness at the edges of graphene nanoribbons. This roughness occurs naturally in graphite samples, as has been seen in scanning tunneling microscopy [18], leading to the localization of charge at the edges and the formation of electronic puddles [19]. As depicted in Fig. 1, disorder at the edges of a graphene ribbon also leads to the formation of "necks", causing an abrupt reduction in the number of conducting channels and thus to a large increase in the impedance along the graphene sheet. This results in the electric isolation of nanoscale size regions, or "dots", where the electrons become temporally confined. Within this picture, Coulomb blockade [20, 21, 22, 23, 24] results from the electron transport from dot to dot through graphene necks.

In our analysis, we will follow the standard theory of Coulomb blockade effects, neglecting the electronic level spacing within the grains. Note that graphene states delocalized throughout a region of linear size $W$ show level spacings of order $\hbar v_{\mathrm{F}} / W$, which is the same scaling behavior followed by the charging energy of a grain of size $W, e^{2} / W$. It seems likely, however, that the rough edges of the samples studied in [17], as well as the internal lattice defects, can lead to a variety of partly or fully localized states at energies close to the Fermi level, reducing the electronic level spacing [4, 25, 26, 27].

In the presence of Coulomb blockade characterized by a renormalized charging energy $E_{c}^{*}$ (which here we assimilate to $E_{g}$ ), the conductance between neighboring metallic dots is renormalized to lower values as the energy or temperature scale is reduced [28, 29, 30]. This 


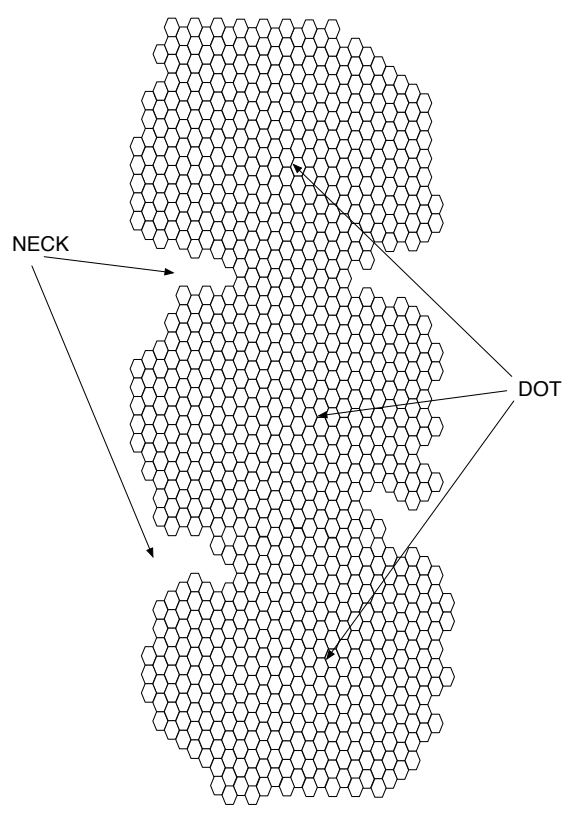

FIG. 1: Illustration of a graphene ribbon with a disordered edge leading to the formation of necks and dots along the ribbon. Coulomb blockade takes place when the charge moves from dot to dot.

renormalization begins to be appreciable at temperatures $T \lesssim E_{c} / k_{B}$, where $E_{c}$ is the charging energy associated to the geometric capacitance of the dot. A perturbative analysis, valid for relatively high temperatures such that $T \sim E_{c} / k_{B}$, leads to a conductance given by:

$$
G=G_{0}-G_{Q} c \ln \left(\frac{E_{c}}{k_{B} T}\right),
$$

where $c=2$ and $G_{0}=4 G_{Q} N_{\mathrm{ch}} \tau$ is the non-interacting conductance (we include spin and valley degeneracy), $G_{Q}=e^{2} / h=38.7 \mu \mathrm{S}$ being the quantum of conductance, $N_{\mathrm{ch}}=k_{F} W / \pi$ the number of transverse orbital channels, and $\tau$ the average transmission per channel. Eq. (11) is valid when the transmission per channel is low, $\tau \ll 1$, although the total conductance can take arbitrary values. A similar expression can be obtained in the limit $\tau \lesssim 1$, provided that none of the channels has perfect transmission [31]. In the latter case, the constant $c$ in Eq. (1) takes the value $c=8 / \pi^{2}$. Within the relatively narrow range of widths and temperatures considered in Ref. 17], the logarithm in Eq. (11) can be taken as a constant of order unity, leading to the approximate expression:

$$
G \simeq\left(4 G_{Q} k_{F} \tau / \pi\right)\left(W-W_{0}\right)
$$

where $W_{0} \approx \pi c / 4 k_{F} \tau$.

The experimental dependence of $G$ on $W$ can be used to estimate the average transmission $\tau$. Figure 2 of Ref. 17] indicates that, at room temperature, and for a gate voltage $V_{g}-V_{\text {Dirac }}=-50 \mathrm{~V}$, a change in conductance $\Delta G \approx 80 \mu \mathrm{S}$ takes place if the width changes by $\Delta W \approx 40 \mathrm{~nm}$. This yields $W_{0} \approx 40 \mathrm{~nm}$. The corresponding hole density in this experiment is $n=3.6 \times 10^{12}$ $\mathrm{cm}^{-2}$, which implies that $k_{\mathrm{F}}^{-1}=1 / \pi \sqrt{n} \approx 1.7 \mathrm{~nm}$. These experimental results are consistent with Eq. (2) if the average transmission per channel is $\tau \approx 0.07$. The total number of channels, for a width $W \approx 40 \mathrm{~nm}$, is $4 N_{\mathrm{ch}} \approx 4 k_{\mathrm{F}} W / \pi \approx 30$. The average transmission probability found here is consistent with tight-binding calculations for wedge shaped graphene constrictions [32] (see also [33, 34]).

The analysis which at high temperatures leads to Eq. (11) also shows that, at low temperatures, the effective charging energy is renormalized by virtual jumps of the electrons across the junction, leading to:

$$
\begin{aligned}
E_{g} & \approx E_{c} e^{-G / c G_{Q}} \\
& =E_{c} e^{-4 N_{\mathrm{ch}} \tau / c}=E_{c} e^{-4 k_{\mathrm{F}} W \tau / \pi c},
\end{aligned}
$$

where $E_{c}$ is the charging energy for the completely isolated dot. It seems reasonable to assume that $E_{c}$ is determined by the nominal ribbon width $W, E_{c} \sim e^{2} / W$ since that is the size of a typical puddle which is isolated from the rest of the ribbon through a contact (see Fig. 1). This contact acts as the bottleneck which determines the conductance of the graphene nanoribbon. This fact does not preclude, however, the possibility of further structure in the I-V characteristic which could be induced by the presence of other dots.

The capacitance of the grains is also modified by the presence of metallic leads and gates. The leads, and the regions of the quasi-one-dimensional ribbon at distances greater than $W$ from the island considered do not change appreciably the charging energy, as a onedimensional charge distribution does not screen an electrostatic potential. The screening of a metallic gate at a distance $d$ from the island can be analyzed, when $d \gg W$, by assuming that the charge in the island induces an image charge. The charging energy is changed to $E_{c} \sim e^{2} / W-e^{2} / 2 d$. In the following, we neglect the second term, as $W \sim 20-100 \mathrm{~nm}$ and typical distances to the gate [1] are $d \sim 300 \mathrm{~nm}$. Charging effects should be strongly suppressed when the distance to the metallic gate is comparable, or smaller, than the width of the ribbon.

Finally, we have identified the value of the Coulomb gap at low temperatures with the gap in the I-V characteristics measured in Ref. [17]. Note that the argument in the exponent in Eq. (3) becomes $4 k_{\mathrm{F}} W \tau / \pi c \approx 0.8$ for $W \approx 40 \mathrm{~nm}$, which leads to an appreciable renormalization of the geometrical charging energy by virtual charge fluctuations (see [35] for similar effects in a different granular system). We note that $E_{c} \sim 4 \mathrm{meV}$ for $W \approx 40 \mathrm{~nm}$.

We can also write Eq. (3) as:

$$
E_{g}(W) \approx \frac{e^{2}}{W} e^{-W / W_{0}}
$$




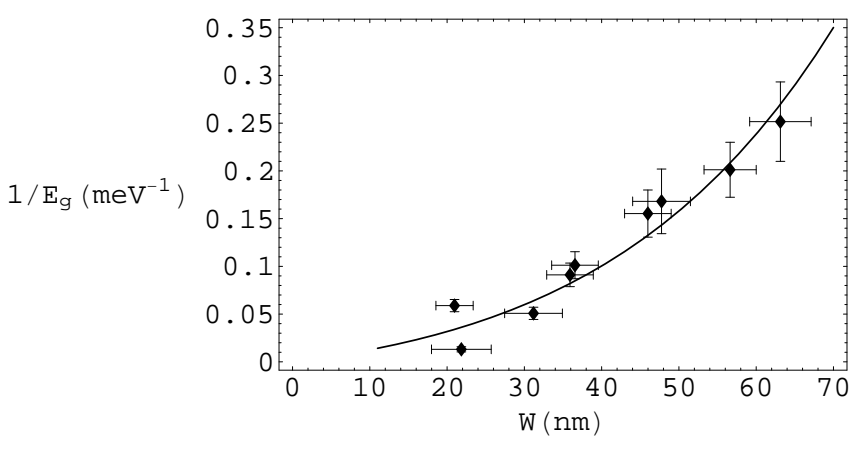

FIG. 2: Comparison between experimental data of Ref. 17] and the theoretical result (full line): $E_{g}^{-1}=B W e^{C W}$, obtained from Eq. (4) with $B=0.001(\mathrm{meV} \times \mathrm{nm})^{-1}$ and $C=0.023 \mathrm{~nm}^{-1}$.

where $W_{0}$ is the length scale used in Eq. (2). Thus, as a function of $W, E_{g}^{-1}$ takes low values for $W \ll W_{0}$ while experiencing a sharp rise for $W \gtrsim W_{0}$. We note that, $E_{g}^{-1}\left(W_{0}\right) \approx 5 \times 10^{-3}(\mathrm{meV})^{-1}$, substantially smaller that the unit scale used in Fig. 3e of Ref. [17]. One can see from Fig. 2 that Eq. (4) explains the experimental data of Ref. 17] within its error bars. A comparison between the expression $E_{g}^{-1}=B W e^{C W}$, obtained from Eq. (4) and the data in [17] yields $C^{-1}=43 \mathrm{~nm}$, which is in good agreement with our theoretical estimate for $W_{0}$. One also obtains $B=10^{-3}(\mathrm{meV} \times \mathrm{nm})^{-1}$, which agrees reasonably with $e^{-2}=6.95 \times 10^{-4}(\mathrm{meV} \times \mathrm{nm})^{-1}$. The fact that $B^{-1}$ is smaller than $e^{2}$ can be partly attributed to the screening effect of the gate.

Finally, we note some trends which provide additional qualitative support to the Coulomb blockade picture. Figs. 3a, 3c, and 3d of Ref. [17] show the differential conductance as a function of the bias and gate voltage. Electron-hole symmetry explains the symmetric behavior around a value of $V_{g}$ which must be identified with the neutrality point. The maximum vertical width of the dark (low differential conductance) zone must be identified with the gap $E_{g}(W)$, which clearly decreases with $W$, in qualitative agreement with Eq. (4). On the other hand, varying $V_{g}$ is equivalent to changing $k_{\mathrm{F}}$. Eq. (3) shows that the variation of the gap and the differential conductance as a function of $k_{\mathrm{F}}$ must be faster for large values of $W$, in good agreement with the experimental results.

The experiment of Ref. [17] also shows that the linear $\left(V_{b} \rightarrow 0\right)$ conductance depends weakly on the gate voltage at the neutrality point, where $k_{\mathrm{F}} \rightarrow 0$. Our analysis predicts that the linear conductance depends on gate voltage through the product $k_{\mathrm{F}} \tau$. The well-known existence of a minimum in the bulk conductivity can be translated, within a Drude picture, into $k_{\mathrm{F}} \tau$ tending to a constant value as $k_{\mathrm{F}} \rightarrow 0$. This trend is consistent with the experimental observation described above. The insensitivity of the conductance to the gate voltage can also be expected to occur when puddles are formed with a finite (positive or negative) charge density [36, 37, 38].

The analysis presented so far describes the observed low temperature gap in transport measurements in terms of the features of isolated junctions, neglecting effects associated to interference effects between multiple junctions. We do not expect these effects to change significantly the analysis. Coulomb blockade leads to the suppression of phase coherence between successive tunneling events. The scaling equation (11) is valid for granular arrays, and transport gaps in these systems have the same functional dependence as in single grains 39]. Hence, in a disordered system the transport properties will be dominated by the junctions with the highest gaps. Note also that inelastic cotunneling processes [40, 41], which influence the conductance of single junctions at low temperatures, and which are not considered here, are strongly suppressed in junction arrays.

In conclusion, we find that the gaps observed in conductance measurements on graphene nanoribbons in Ref. [17] can be explained as Coulomb gaps due to the existence of internal junctions between graphene islands, where the transmission, for all transverse channels, is less than one. We identify the gaps observed in transport measurements as the effective charging energy of the islands, renormalized by the charge fluctuations at the junctions. We further simplify the model by assuming that the transport properties can be studied by analyzing a single representative junction. The model leads to a simple dependence of the gaps on material parameters, such as the carrier concentration, or the width of the ribbons. We obtain a reasonable agreement with the experiments.

Charging effects are mostly determined by the geometry of the system and by the amount of screening of the Coulomb interaction. The explanation proposed here implies that the gaps observed in transport measurements should be weakly affected by static disorder, or by changes in the electron interference properties, such as those induced by an applied magnetic field. On the other hand, we expect that charging effects should be suppressed by metallic gates at distances from the ribbon which are smaller than the ribbon width.

We are thankful to A. K. Geim, P. Kim, I. Martin, V. M. Pereira, and J. J. Palacios, for helpful discussions. This work has been supported by MEC (Spain) under Grants FIS2004-05120, FIS2005-05478-C02-01, the EU Contract 12881 (NEST), the EU Marie Curie RTN Programme no. MRTN-CT-2003-504574, and the Comunidad de Madrid program CITECNOMIK, no. CM2006S-0505-ESP-0337. AHCN is supported through NSF grant DMR-0343790. 
[1] K. S. Novoselov, A. K. Geim, S. V. Morozov, D. Jiang, Y. Zhang, S. V. Dubonos, I. V. Grigorieva, and A. A. Firsov, Science 306, 666 (2004).

[2] A. H. Castro Neto, F. Guinea, and N. M. R. Peres, Physics World 19, 33 (2006)

[3] M. I. Katsnelson, K. S. Novoselov, and A. K. Geim, Nature Physics 2, 620 (2006).

[4] K. Nakada, M. Fujita, G. Dresselhaus, and M. S. Dresselhaus, Phys. Rev. B 54, 17954 (1996).

[5] C. Berger, Z. M. Song, X. B. Li, X. S. Wu, N. Brown, C. Naud, D. Mayo, T. B. Li, J. Hass, A. N. Marchenkov, E. H. Conrad, P. N. First, and W. A. de Heer, Science 312, 5777 (2006).

[6] A. K. Geim, and K. S. Novoselov, Nature Materials 6, 183 (2007).

[7] J. González, F. Guinea, and M. A. H. Vozmediano, Phys. Rev. Lett. 69, 172 (1992).

[8] N. M. R. Peres, F. Guinea, and A. H. Castro Neto, Phys. Rev. B 73, 125411 (2006).

[9] Y. Zhang, Z. Jiang, J. P. Small, M. S. Purewal, Y. W. Tan, M. Fazlollahi, J. D. Chudow, J. A. Jaszczak, H. L. Stormer, and P. Kim, Phys. Rev. Lett. 96, 136806 (2006).

[10] K. Nomura, and A. H. MacDonald, Phys. Rev. Lett. 96, 256602 (2006).

[11] M. O. Goerbig, R. Moessner, and B. Doucot, Phys. Rev. B 74, 161407 (R) (2006)

[12] P. R. Wallace, Phys. Rev. 71, 622 (1947).

[13] K. W.-K. Shung, Phys. Rev. B 34, 979 (1986).

[14] B. Wunsch, T. Stauber, F. Sols, and F. Guinea, New J. Phys. 8, 318 (2006).

[15] S. Das Sarma, E. H. Hwang, W.-K. Tse, Phys. Rev. B 75, 121406 (R) (2007).

[16] Y. Barlas, T. Pereg-Barnea, M. Polini, R. Asgari, and A. H. MacDonald, cond-mat/0701257, M. Polini, R. Asgari, Y. Barlas, T. Pereg-Barnea, and A. H. MacDonald, arXiv:0704.3786

[17] M. Y. Han, B. Özyilmaz, Y. Zhang, and P. Kim, Phys. Rev. Lett. 98, 206805 (2007).

[18] Y. Kobayashi, K. Fukui, T. Enoki, K. Kusakabe, and Y. Kaburagi, Phys. Rev. B 71, 193406 (2005).

[19] A. H. Castro Neto, F. Guinea, and N. M. R. Peres, Phys.
Rev. B 73, 205408 (2006).

[20] E. Ben-Jacob, E. Mottola and G. Schön, Phys. Rev. Lett. 51, 2064 (1983).

[21] T.-L. Ho, Phys. Rev. Lett. 51, 2060 (1983).

[22] E. Ben-Jacob, and Y. Gefen, Physics Letters 108A, 289 (1985).

[23] D. V. Averin and K. K. Likharev, in Mesoscopic Phenomena in Solids, B. L. Altshuler, P. A. Lee and R. A. Webb, eds. (Elsevier, Amsterdam, 1991).

[24] H. Grabert and M. H. Devoret, eds., Single Electron Tunneling (Plenum, New York, 1992).

[25] M. Fujita, K. Wakabayashi, Nakada, and K. Kusakabe, J. Phys. Soc. Jpn. 65, 1920 (1996).

[26] M. A. H. Vozmediano, M. P. López-Sancho, T. Stauber, and F. Guinea, Phys. Rev. B 72, 155121 (2005).

[27] V. M. Pereira, F. Guinea, J. M. B. Lopes dos Santos, N. M. R. Peres, A. H. Castro Neto, Phys. Rev. Lett. 96, 036801 (2006).

[28] F. Guinea, and G. Schön, Europhys. Lett. 1, 585 (1986).

[29] G. Schön, and A. D. Zaikin, Phys. Rep. 198, 237 (1990).

[30] C. Herrero, A. D. Zaikin, and G. Schön, Phys. Rev. B 59, 5728 (1999).

[31] Y. V. Nazarov, Phys. Rev. Lett. 82, 1245 (1999).

[32] F. Muñoz-Rojas, D. Jacob, J. Fernández-Rossier, and J. J. Palacios, Phys. Rev. B 74, 195417 (2006).

[33] E. Louis, J. A. Vergés, F. Guinea, and G. Chiappe, Phys. Rev. B 75, 085440 (2007).

[34] I. Martin, and Ya. M. Blanter, arXiv:0705.0532,

[35] M. García-Hernández, F. Guinea, A. de Andrés, J. L. Martínez, C. Prieto, and L. Vázquez, Phys. Rev. B 61, 9549 (2000).

[36] A. H. Castro Neto and Eun-Ah Kim, cond-mat/0702562

[37] J. Martin, N. Akerman, G. Ulbricht, T. Lohmann, J. H. Smet, K. von Klitzing, and A. Yacoby, arXiv:0705.2180.

[38] F. Guinea, M. I. Katsnelson, and M. A. H. Vozmediano, arXiv:0707.0682

[39] D. P. Arovas, F. Guinea, C. P. Herrero, and P. San José, Phys. Rev. B 68, 085306 (2003).

[40] D. V. Averin, and Y. V. Nazarov, Phys. Rev. Lett. 65, 2446 (1990).

[41] L. I. Glazman, and K. A. Matveev, Sov. Phys. JETP Lett, 51, 484 (1990). 